\title{
A wireless infrared sensor network for the estimation of the position and orientation of a moving target
}

\author{
Nikos Petrellis, Nikos Konofaos and George Alexiou \\ Computer Engineering and Informatics Dept. \\ University of Patras \\ Rio Campus, Patras, GREECE \\ $+302610996933$ \\ petrelis@ceid.upatras.gr
}

\begin{abstract}
The location of a moving person or vehicle in a virtual reality environment is a critical issue. A wireless infrared sensor network capable of estimating the position of a target on a plane and its orientation is presented in this paper. This is actually an extension of the position estimation system that was presented in [1-2] where the target was allowed to move on a plane but not to rotate. In [1-2] the estimation of the position was based on a network consisting of a few low cost infrared transmitters and a pair of infrared receivers mounted on the target. In this paper we discuss how a third infrared sensor properly positioned at the side of the receiver can also allow the estimation of the target orientation.
\end{abstract}

\section{Keywords}

Position localization, infrared sensors, wireless sensor networks.

\section{INTRODUCTION}

Several approaches that have been proposed in the past, for target localization in automation and robotics are based on different types of media. The dominant methods rely on measuring the RF or ultrasound signal strength of multiple transmitting devices [3] or the time of the fly of short (laser, infrared) signal pulses reflected by the surface of the object under measurement [4-7]. Both of these approaches require high quality analog sensors and fast processing units capable of measuring accurately very short time intervals. In robotics and virtual reality applications this procedure is assisted by additional methods like image processing, odometers, accelerometers etc [8-10]. The architectural and computational cost of these approaches is significant.

We have recently exploited the quality of the received infrared patterns in order to estimate the distance of the receiver from the transmitter. More specifically, we have shown [2] that the position of a moving target on a grid plane of $15 \mathrm{~m}^{2}$ can be estimated if two infrared pattern transmitters are positioned at the borders of the covered area and a pair of sensors is mounted in opposite

Permission to make digital or hard copies of all or part of this work for personal or classroom use is granted without fee provided that copies are not made or distributed for profit or commercial advantage and that copies bear this notice and the full citation on the first page. To copy otherwise, to republish, to post on servers or to redistribute to lists, requires prior specific permission and/or a fee. MOBIMEDIA 2007, August 27-29, Nafpaktos, Greece

Copyright @ 92007 ICST 978-963-06-2670-5

DOI 10.4108/ICST.MOBIMEDIA2007.1584 directions at the side of the target. The area covered can be extended if multiple transmitters are used. Nevertheless, it is essential that the target does not rotate in order to predict its position accurately.

In the present work, we discuss the way a third sensor at the side of the receiver can resolve the aforementioned limitation. The position estimation history is also used in order to increase the precision of the estimation and extend the covered area when multiple infrared pattern transmitters are used.

In Section 2 we briefly describe the architecture of the system described in [1-2] and present the extensions added in the current work. In Section 3 we explain the position and orientation estimation method that is demonstrated by a simple case study.

\section{SYSTEM ARCHITECTURE}

The position localization system presented in [1-2] consists of the infrared transmitting devices IRTX1 and IRTX2 that are placed around the covered area as shown in Fig. 1. These devices transmit different sets of patterns that are received with varying quality by the pair of receivers IRRX $\mathrm{I}_{\mathrm{A}}$ and $\mathrm{IRRX}_{\mathrm{B}}$ at the side of the target. The reception quality is defined as the number of the received patterns of a specific type compared to the expected ones in a specific time interval (success rate of this pattern). The system cost is drastically reduced using this method since we merely count digital patterns instead of analog signal strength or very short time intervals. Each one of the transmitting devices supports a set $R$ of pattern types ( $R 1$ for IRTX1 and $R 2$ for IRTX2). There are no common pattern types in these sets $(R 1 \cap R 2=\emptyset)$. The pattern types differ in the number of pulses they consist of and their duration. A pattern type MOD $i$ consists of $i$ pulses. If $i>j$ then MOD $j$ will be received with a higher success rate than MOD $i$ at the same position since it consists of smaller number of pulses (there is higher probability to receive a pulse distorted in MOD $i$ than MOD $j$ ). The pulse durations of MOD $i$ are defined shorter than those of MOD $j$ in order to further strengthen the previous assumption.

Before real time operation, the target visits the nodes of a virtual grid that covers the area around which the target moves and records the success rates of the various patterns received (calibration stage). The set of these rates define a unique identity for each grid node on the plane. During real time operation the retrieved success rates at the current position are compared to the identities stored from the calibration stage and the closest node is selected. Then, an interpolation search in two dimensions is 
carried out in order to further approximate the real target position. If the target is allowed to rotate around himself, the position estimation method would be mislead since the success rates measured at the same position by $\operatorname{IRRX}_{\mathrm{A}}$ and $\operatorname{IRRX}_{\mathrm{B}}$ would be different for different target orientation.

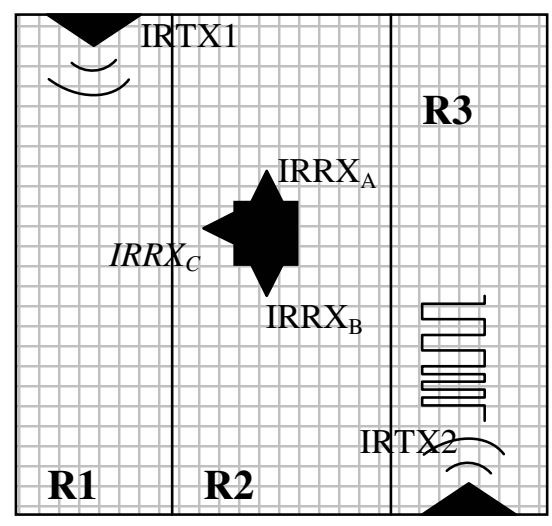

Figure 1. The architecture and topology of the position localization system.

In the present work, a third IRRX device $\left(\operatorname{IRRX}_{\mathrm{C}}\right)$ is connected to the processing unit of the target in order to distinguish between a target move and a target rotation. The third IRRX device actually increases the identities dimensions in order to make the monitoring of both the motion and the rotation of the target feasible. Moreover, the indications retrieved from previous observations are used in order to evaluate the current localization results. In [2] we presented the single state machine that is used at the processing unit and controls the two IRRX devices. It would be quite complicated if we attempted to control three IRRX devices using a single state machine. Hence, we modified the processing unit software in order to implement three individual state machines fed by the same event queue and their corresponding processes. An input monitoring process controls the IRRX devices and feeds a FIFO queue with three types of events: preamble $\left(\mathrm{PRE}_{\mathrm{A}}, \mathrm{PRE}_{\mathrm{B}}, \mathrm{PRE}_{\mathrm{C}}\right)$, pulse $\left(\mathrm{PLS}_{\mathrm{A}}, \mathrm{PLS}_{\mathrm{B}}\right.$, $\mathrm{PLS}_{\mathrm{C}}$ ) and pause $\left(\mathrm{PAUS}_{\mathrm{A}}, \mathrm{PAUS}_{\mathrm{B}}, \mathrm{PAUS}_{\mathrm{C}}\right.$ ). If a low cost 8-bit microcontroller is used as a processing unit, the three state machine processes can be implemented as subroutines called in turn by an infinite loop, while the input monitoring process may be implemented as an interrupt service routine that is called whenever the level changes on an IRRX pin.

In Fig. 2, the state machine of IRRX $\mathrm{A}_{\mathrm{A}}$ is presented. At power up it enters the state NO_PREAM ${ }_{A}$ and waits for a preamble $\left(\mathrm{PR}_{\mathrm{A}}\right.$ event) in order to enter the RCVD_PRE $E_{A}$ state. In this state the pulses arriving at $\operatorname{IRRX}_{\mathrm{A}}$ are accumulated in the variable $T$ until a pause interval is observed ( PAUS $_{\mathrm{A}}$ event). The current value of $T$ indicates that the MODT pattern was recognized. The number of patterns recognized from each $\mathrm{MOD} i$ type are stored in the corresponding position of the vector $A(A[i])$. When a preamble is recognized, the vector $A$ is sent to a host computer through a serial port. Two more state machines similar to the one presented in Fig. 2 are defined in order to store the success rates retrieved from IRRX $\mathrm{B}_{\mathrm{B}}$ and IRRX $\mathrm{I}_{\mathrm{C}}$ in the vectors $B$ and $C$ respectively. The values of the vectors $A, B$ and $C$ are used at the host computer in order to estimate the position of the target using the method described in the next section. The automation, virtual reality or ubiquitous computing application that uses the position estimation results is also installed on the host computer.

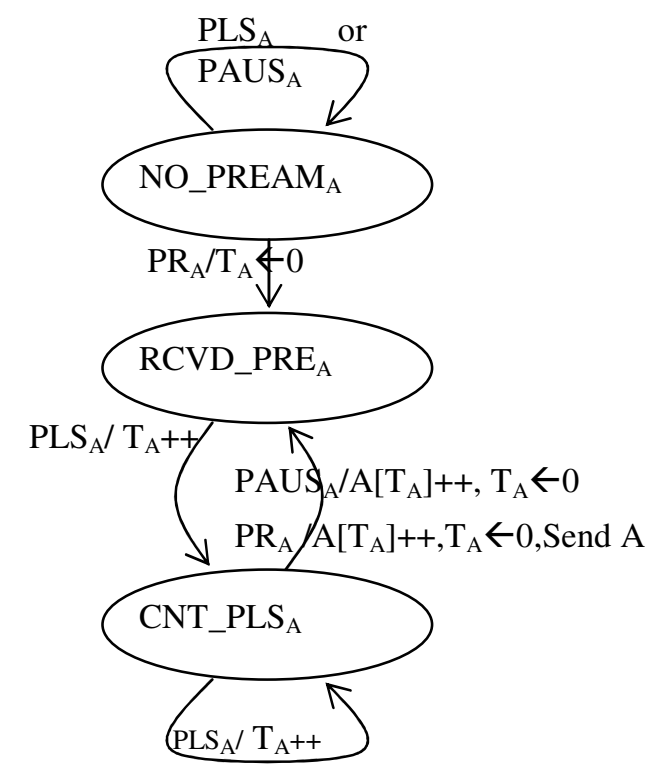

Figure 2. The state machine for the $\operatorname{IRRX}_{\mathrm{A}}$ device.

\section{ESTIMATION OF THE POSITION AND ORIENTATION OF A TARGET}

If the infrared patterns reflections are negligible inside the covered area, the success rate that is measured by an IRRX device changes smoothly with respect to the orientation of the target and its distance from an IRTX transmitter. If we focus on the orientation of the target we may use the curves of Fig. 3 where the success rates of MOD2, MOD5, MOD6 and MOD9 are drawn as a function of the angle for a specific distance $(2.5 \mathrm{~m})$ from an IRTX device. The specific IRTX device of Fig. 3 has a single IR emitting diode (SFH485) biased for moderate power consumption $(20 \mathrm{~mW})$. As we see, at that specific distance, the MOD9 patterns cannot be received at all. The rest of the patterns are received with a maximum success rate when the IRRX faces towards the IRTX device $\left(0^{\circ}\right.$ angle $)$. After studying a number of curves similar to the one presented in Fig. 3 for various distances, we can construct Table 1 for the three major regions designated in Fig. 1 (R1, R2, R3) and for 4 possible target orientations. Hence, $\mathrm{O} 1$ is the orientation presented in Fig. 1, while $\mathrm{O} 2, \mathrm{O} 3$ and $\mathrm{O} 4$ are derived if the target is rotated clockwise by $90^{\circ}, 180^{\circ}$ and $270^{\circ}$ respectively.

In order to describe the quality of the pattern reception, we use the notation used in Table 1: number-qualification. The number denotes the IRTX patterns received ( 1 or 2 if the patterns were transmitted by IRTX1 or IRTX2 respectively) while the qualification may take the values $\mathrm{H}$ (if success rate $>5$ ), $\mathrm{L}$ (success rate between 1 and 5) and VL (success rate $<1$ ). 


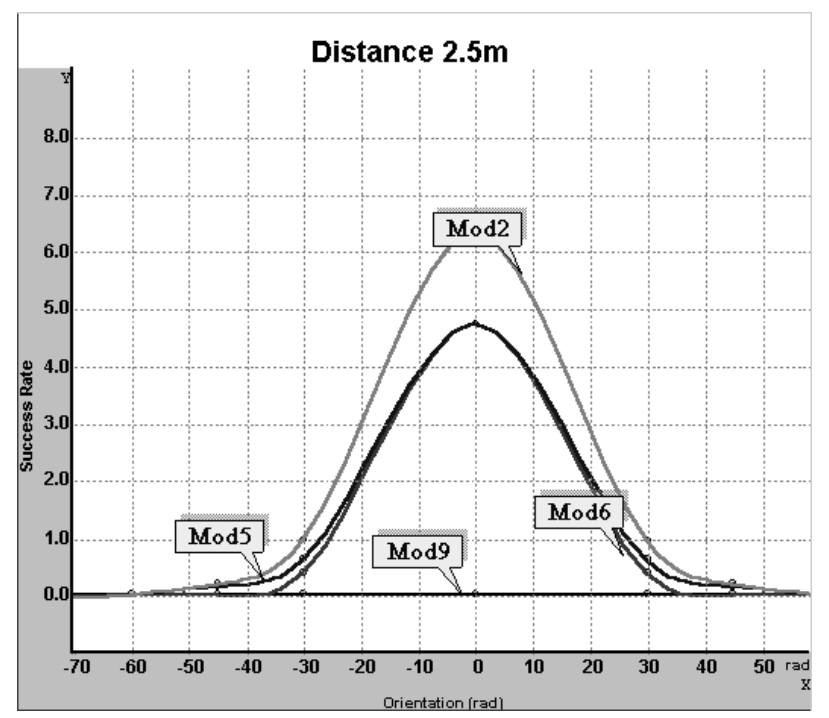

Figure 3. The Success Rate of 4 pattern types as a function of the IRRX orientation.

Table 1. Quality of pattern reception.

\begin{tabular}{|l|l|l|l|l|}
\hline Orient. & IRRX & R1 & R2 & R3 \\
\hline \multirow{4}{*}{ O1 } & IRRX $_{\mathrm{A}}$ & $1 \mathrm{H}$ & $1 \mathrm{H}$ & $1 \mathrm{~L}$ \\
\cline { 2 - 5 } & IRRX $_{\mathrm{B}}$ & $2 \mathrm{~L}$ & $2 \mathrm{H}$ & $2 \mathrm{H}$ \\
\cline { 2 - 5 } & IRRX $_{\mathrm{C}}$ & $1 \mathrm{VL}$ & $1 \mathrm{~L}$ & $1 \mathrm{~L}, 2 \mathrm{~L}$ \\
\hline O2 & IRRX $_{\mathrm{A}}$ & $1 \mathrm{~L}, 2 \mathrm{~L}$ & $2 \mathrm{~L}$ & $2 \mathrm{VL}$ \\
\cline { 2 - 5 } & IRRX $_{\mathrm{B}}$ & $1 \mathrm{VL}$ & $1 \mathrm{~L}$ & $1 \mathrm{~L}$ \\
\cline { 2 - 5 } & IRRX $_{\mathrm{C}}$ & $1 \mathrm{H}$ & $1 \mathrm{H}$ & $1 \mathrm{~L}$ \\
\hline O3 & IRRX $_{\mathrm{A}}$ & $2 \mathrm{~L}$ & $2 \mathrm{H}$ & $2 \mathrm{H}$ \\
\cline { 2 - 5 } & IRRX $_{\mathrm{B}}$ & $1 \mathrm{H}$ & $1 \mathrm{H}$ & $1 \mathrm{~L}$ \\
\cline { 2 - 5 } & IRRX $_{\mathrm{C}}$ & $2 \mathrm{~L}$ & $2 \mathrm{~L}$ & $2 \mathrm{VL}$ \\
\hline \multirow{3}{*}{ O4 } & IRRX $_{\mathrm{A}}$ & $1 \mathrm{~L}$ & $1 \mathrm{~L}$ & $1 \mathrm{~L}, 2 \mathrm{~L}$ \\
\cline { 2 - 5 } & IRRX $_{\mathrm{B}}$ & $1 \mathrm{~L}, 2 \mathrm{~L}$ & $2 \mathrm{~L}$ & $2 \mathrm{~L}$ \\
\cline { 2 - 5 } & IRRX $_{\mathrm{C}}$ & $2 \mathrm{~L}$ & $2 \mathrm{H}$ & $2 \mathrm{H}$ \\
\hline
\end{tabular}

The position estimation method may have several levels of complexity according to the desired accuracy. If the position estimation method is simply used to provide an indication of whether the target resides within a rough region (like $R 1, R 2$ or $R 3$ in Fig. 1), then Table 1 can be directly accessed in order to locate the pair (region,orientation).

The area covered can be extended if more than two IRTX devices are placed as shown in Fig. 4. If the system keeps track of the regions that the target visits, it is easy to locate his position although some of the indications are identical in multiple regions. For example, the dashed line in Fig. 4 shows the track of the target that has crossed the regions R1, R2, R3, R2, R3', R1' and $\mathrm{R} 2$ in this turn. R3' would be identical to R3 if the presented topology was part of a wider area with a large number of alternating IRTX1 and IRTX2 devices. Similarly, R1 would be identical to R1' if an IRTX2 device was placed at the left of R1 as is the case in R1'. Nevertheless R2 appears twice in the presented area but the $\mathrm{R} 2$ region at the right is assigned different coordinates and the localization system concludes that the target has entered the right R2 since it has previously crossed R3' and $\mathrm{R} 1$ ' in this turn. In this way, the area can be extended using only two types of IRTX devices.

The designated regions can be split in smaller ones and the draft limits of each region can be defined using tables similar to Table 1. A necessary requirement is to define more than the 3 success rate ranges $(\mathrm{H}, \mathrm{L}, \mathrm{VL})$ that were used for the proof of concept in this paper. It has been experimentally tested that a $3 \mathrm{mX} 3 \mathrm{~m}$ area covered by two IRTX devices can be split in up to 36 regions of $50 \mathrm{cmX} 50 \mathrm{~cm}$ that can be identified using the simple method described above.

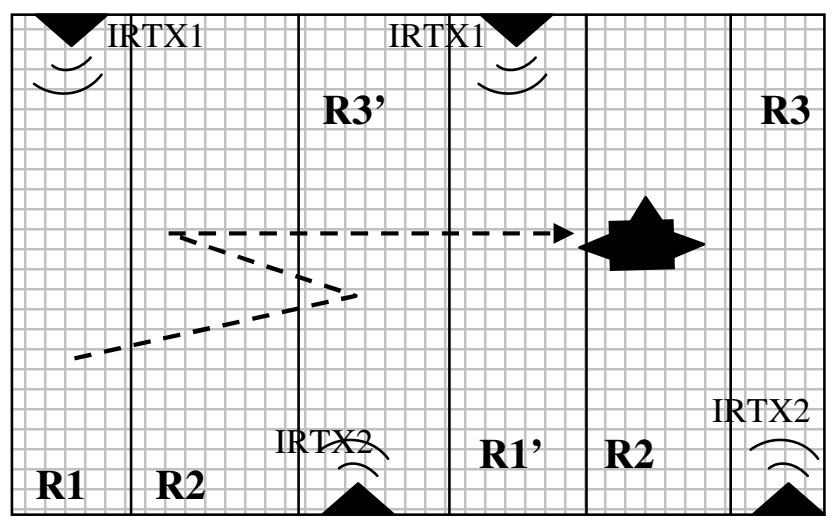

Figure 4. Extension of the covered area.

If a position/orientation estimation method with higher precision is required and the reflections are not negligible, an approach like the one presented in [2] must be followed. More specifically, a calibration stage would be required before real time operation, in order to familiarize the target with the environment. During the calibration stage, the target visits the nodes of a virtual grid and records the set of the $A, B$ and $C$ vectors for a number of orientation steps depending on the desired accuracy. For example, if the desired orientation accuracy is $30^{\circ}$ then 12 vector sets should be retrieved for each grid node.

At real time operation, the set of the success rate vectors retrieved at the current position is compared to all calibration vector sets and as an outcome, the pair (node, orientation) that is closer to the current values is selected. The comparison is based on the following equations [2]:

$$
\begin{aligned}
& d_{i k j}=\left\{\begin{array}{l}
\frac{v_{j}-r_{i k j}}{v_{j}}, \text { if }\left(v_{j}>r_{i k j}\right) \\
\frac{r_{i k j}-v_{j}}{r_{i k j}}, \text { if }\left(v_{j} \leq r_{i k j}\right) \text { and }\left(r_{i k j} \neq 0\right) \\
1, i f\left(v_{j}=0\right) \text { or }\left(r_{i k j}=0\right)
\end{array}\right\} \\
& D_{i, k}=\sum_{j} d_{i k j}
\end{aligned}
$$


The parameter $d_{i k j}$ compares the relative distance of a value in one of the currently retrieved vectors $A, B$ and $C$ with the corresponding value in the vectors of the orientation $k$ of the node $j$. If the $d_{i k j}$ parameters of all the positions of the vectors $A, B$ and $C$ are summed up into $\mathrm{D}_{i, k}$, then the lowest $\mathrm{D}_{i, k}$ parameter is used to select the pair $(i, k)$ as a (node, orientation) indication.

The accuracy that can be achieved, depends on the grid node distance and the orientation step used during the calibration. Nevertheless, if very small grid node distances and orientation steps are selected, a large number of vector sets must be retrieved during the calibration stage (increased calibration overhead). Moreover, there is an increased possibility that multiple sets of $A$, $B$ and $C$ vectors may contain similar values, hence confusing the position estimation procedure. Choosing $20 \mathrm{~cm}$ as a grid node distance and $45^{\circ}$ as the orientation step leads to a $100 \%$ success in the estimation results in for $1 \mathrm{mX} 1 \mathrm{~m}$ covered area. The number of vector sets that should be retrieved during the calibration stage in this case is 200 .

\section{CONCLUSIONS}

The position estimation system that we had presented in [1-2] was extended in order to track a third degree of freedom of a moving target: its orientation. We discussed how a properly positioned third sensor at the side of the receiver makes the estimation of the target position and orientation feasible. Simple predefined tables can be used in applications where a rough position estimation and orientation is required. If more accurate results are required, a calibration stage is needed in order to familiarize the target with its environment by retrieving sample measurements at various orientation steps on the nodes of a virtual grid. An estimation error of less than $20 \mathrm{~cm}$ in the distance and $45^{\circ}$ in the orientation can be achieved depending on the area covered by two IRTX devices.

In future work, several topologies of the IRTX devices will be tested in order to suggest optimal grid node distances and orientation steps. The refinement step described in [2] may be extended in three dimensions leading to more accurate results without having to adopt shorter grid node distances and orientation steps. Finally, an extension of the position estimation system for 4 degrees of freedom can also be studied.

\section{REFERENCES}

[1] Petrellis, N., Konofaos, N. and Alexiou, G. Target Localization Utilizing the Success Rate in Infrared Pattern Recognition. IEEE Sensors Journal, 6(5), 2006, 1355-1364.

[2] Petrellis, N.,Konofaos, N. and Alexiou, G. Position Estimation on a Grid Based on Infrared Pattern Reception Features. Proceedings of the Int. Workshop on Ubiquitous Computing (IWUC) (The Paphos, The Cyprus, May 2006), 2006, 21-28.

[3] Ladd, A., Bekiris, K., Rudys, A., Kavraki, L. and Wallach, D. Robotics Based Location Sensing Using Wireless Ethernet. Wireless Networks, Vol. 11, No. 1-2, (Jan 2005), 189-204.

[4] Miura, J., Negishi, Y. and Shirai, Y. Adaptive Robot Speed Control by Considering Map and Motion Uncertainty. Elsevier Robotics and Autonomous Systems, 54(2006), 110117.

[5] Victorino A., Rives, P. and Borelly, J. Safe Navigation for Indoor Mobile Robots Part II: Exploration, Self Localisation and Map Building. Int. Journal of Robotics Research. Vol. 22, No. 12 (Dec 2003), 1019-1039.

[6] Bicho, E., Mallet, P. and Schoner, G. Target Representation on an Autonomous Vehicle with Low Level Sensors, Int. Journal of Robotics Research, Vol. 19, No. 5 (May 2000) 424-447.

[7] Holmberg, P. Ultrasonic Sensor Array for Positioning and Rotation Estimates of Planar Surfaces. Sensors and Actuators A, 44(1994) 37-43.

[8] Borenstein, J. Everett, B. and Feng, L. Navigating Mobile Robots: Systems and Techniques, A.K. Peters Ltd Wellesley, MA, 1996.

[9] Jin, T., Lee, J. and Tso, S. A New Space and Time Sensor Fusion Method for Mobile Robot Navigation. Wiley Journal of Robotics Systems, 21(7), (2004) 389-400.

[10] Porta, J. and Krose B. Appearance-based Concurrent Map Building and Localisation. Elsevier Robotics and Autonomous Systems, 54(2006) 159-164. 